\title{
Rapunzel Syndrome and Small Bowel Intussusceptions Due to a Cotton Thread Bezoar: A Case Report
}

\author{
Vineetha Raghu ${ }^{1} \quad$ Anita N. Nagadi ${ }^{1} \quad$ Ashwini Chethan Kumar ${ }^{1}$ \\ ${ }^{1}$ Department of Radiodiagnosis, Columbia Asia Referral Hospital, \\ Yeshwanthpur, Bengaluru, Karnataka, India

\begin{abstract}
Address for correspondence Vineetha Raghu, MD, DNB, Department of Radiology, Columbia Asia Referral Hospital, 26/4, Brigade Gateway, Beside Metro Cash and Carry West, Yeshwanthpur, Bengaluru 560055, Karnataka, India

(e-mail:vineetha.r@columbiaasia.com).
\end{abstract}

J Gastrointestinal Abdominal Radiol ISGAR 2018;1:56-59

\begin{abstract}
Rapunzel syndrome is a rare disease in which a trichobezoar extends into the duodenum or distal small intestine. It is associated with compulsive eating of indigestible substances or pica and is more common in cases of underlying psychiatric disturbances. If left untreated, gastrointestinal bezoars with or without the Rapunzel syndrome may lead to several complications such as ulceration, perforation, peritonitis, pancreatitis, biliary obstruction, severe nutritional disturbances, and rarely intestinal obstruction. We present an exceptionally rare case of Rapunzel syndrome caused by a large cotton thread bezoar in an adolescent girl, associated with multiple jejunal

Keywords

- intussusception

- Rapunzel syndrome intussusceptions. Further, we present a literature review of this entity. An interesting association of bezoar with intussusception is explained. Since a history of pica may not be forthcoming in all cases, it is important to be familiar with the imaging characteristics, complications, and management of bezoars.
\end{abstract}

\section{Introduction}

Compulsive eating disorders or pica can occur in children and adults with psychological disturbances leading to ingestion of indigestible fibers of thread, hair, and other materials. These may subsequently conglomerate and form a mass in the stomach known as a bezoar. When a trichobezoar, made of hair or cotton thread, extends into the duodenum or distal small intestine like a tail, it is referred to as the Rapunzel syndrome, a rare form of trichobezoar., ${ }^{1,2}$

Rapunzel syndrome has usually been described in association with ingested human hair. There have only been a few case reports of cotton thread bezoar reported in the literature, ${ }^{3-5}$ and only rarely reported in association with Rapunzel syndrome. ${ }^{2}$ If undiagnosed, bezoars may lead to gastrointestinal (GI) ulceration, perforation, peritonitis, pancreatitis, biliary obstruction, severe nutritional disturbances, and rarely intestinal obstruction. .,6 $^{5,}$

Intussusception as a complication of bezoar has also been rarely described in the literature, usually associated with bowel obstruction. ${ }^{7,8}$ It has been postulated that the tail of the bezoar and the intraluminal strands interfere with the small bowel peristalsis and hence predispose to intussusception. ${ }^{9}$
It is also possible that a purse-string effect due to shorter length of the bezoar tail, compared with the length of the small intestine, may lead to foreshortening and contraction of the mesenteric side of the small bowel wall, leading to intussusception. ${ }^{10}$ Whatever the cause, it is important to remember that bezoars may sometimes be the undeclared cause of intussusception, especially in recurrent cases.

Imaging is crucial in the diagnosis of Rapunzel syndrome and intussusception. Upper GI endoscopy is the investigation of choice in these patients and may also aid therapeutic retrieval of the bezoar. ${ }^{1}$ We describe a case of Rapunzel syndrome due to a cotton thread bezoar in an adolescent girl associated with multiple small bowel intussusceptions, followed by a review of the literature.

\section{Case Description}

An 11-year-old girl presented to the gastroenterology clinic with complaints of weight loss and loss of appetite, as well as behavioral changes and dullness. There was also a forthcoming history of a craving to eat threads from clothes for nearly 10 years. received

August 6, 2018

accepted after revision

August 18, 2018
License terms

() (1) $\ominus \circledast$ 
On physical examination, a hard, nontender mass was palpated in the epigastrium raining the clinical suspicion of a trichobezoar. Blood tests were normal. A preliminary ultrasonogram of the abdomen and pelvis performed elsewhere demonstrated "excessive bowel gas" and no other significant abnormality. An upper GI endoscopy showed a large gastric trichobezoar entirely filling the gastric lumen and obscuring the distal end of the bezoar. Contrast-enhanced computed tomography (CT) (CECT) of the abdomen was performed on a 128-detector scanner (Philips Ingenuity,

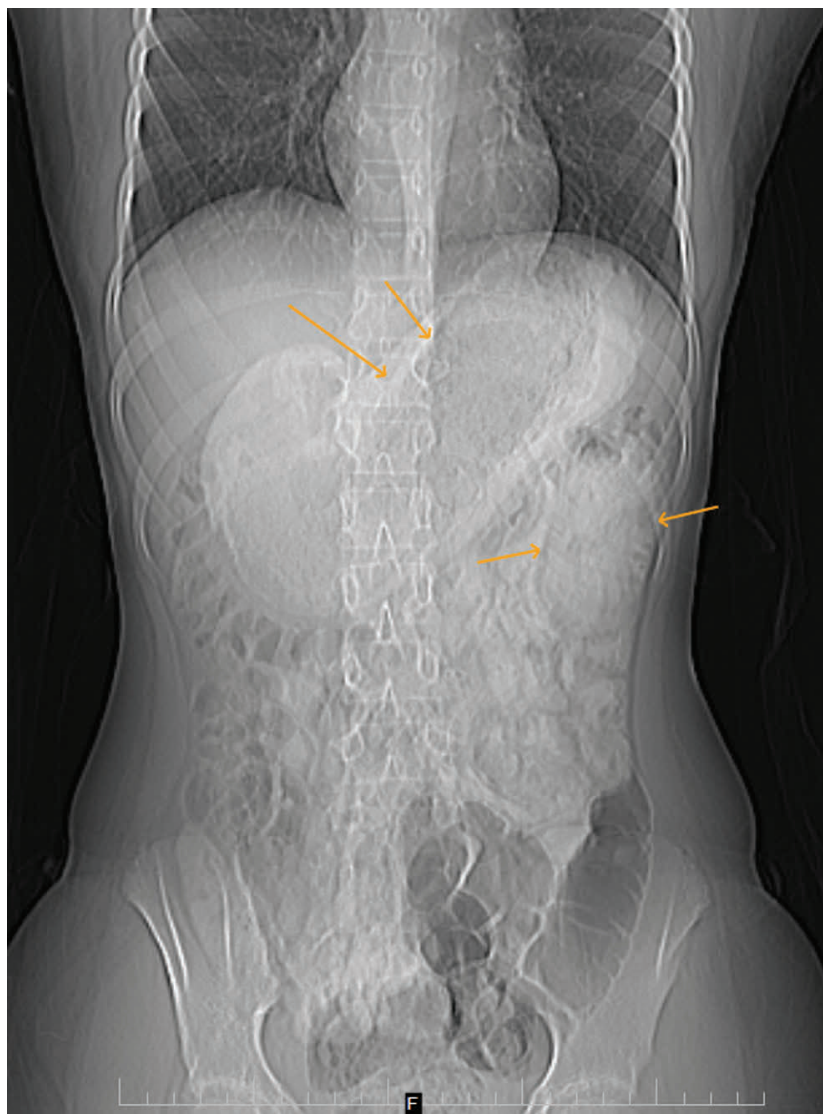

Fig. 1 Topogram demonstrates mottled appearance of an intraluminal bezoar in a mildly dilated stomach and within a dilated jejunal loop in the left upper abdomen (arrows).
Germany) as a single venous phase 70 seconds after an intravenous pressure injection of $45 \mathrm{~mL}$ of Omnipaque (Wipro GE Heathcare Pvt Ltd, Shanghai, China) at $112 \mathrm{mAs}$ and $120 \mathrm{kV}$. CECT demonstrated a large, organized, intraluminal bezoar with a mottled appearance due to entrapped air, extending into the duodenum and proximal jejunum ( - Figs. 1 and 2). The stomach was dilated; there was mild thickening of the stomach and jejunum. At least four short segments of jejunojejunal intussusception were found as "target signs" (-Fig. 3).

Attempts to endoscopically remove the bezoar were futile because the bexoar length was over $25 \mathrm{~cm}$; laparoscopic gastrostomy and removal of the bezoar was planned. The patient, however, refused surgery and was lost to follow-up.

\section{Discussion}

Compulsive eating disorders or pica can occur in children and adults with psychological disturbances leading to ingestion of indigestible fibers of thread, hair, and other materials. These may subsequently conglomerate and form a mass in the stomach known as a bezoar. When a trichobezoar, made of hair or cotton thread, extends into the duodenum or distal

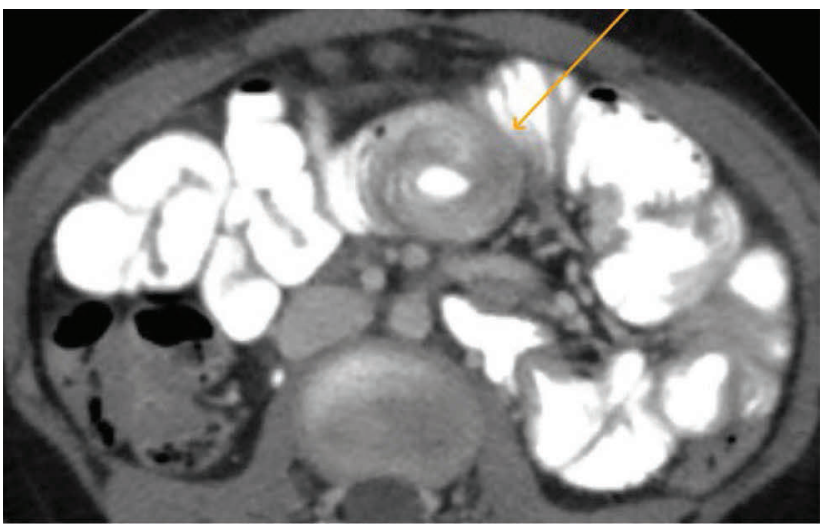

Fig. 3 Axial computed tomography (CT) with oral and intravenous contrast depicts jejunal intussusception (arrow) seen as target or doughnut sign. There is also mild mural thickening of the jejunal loops.
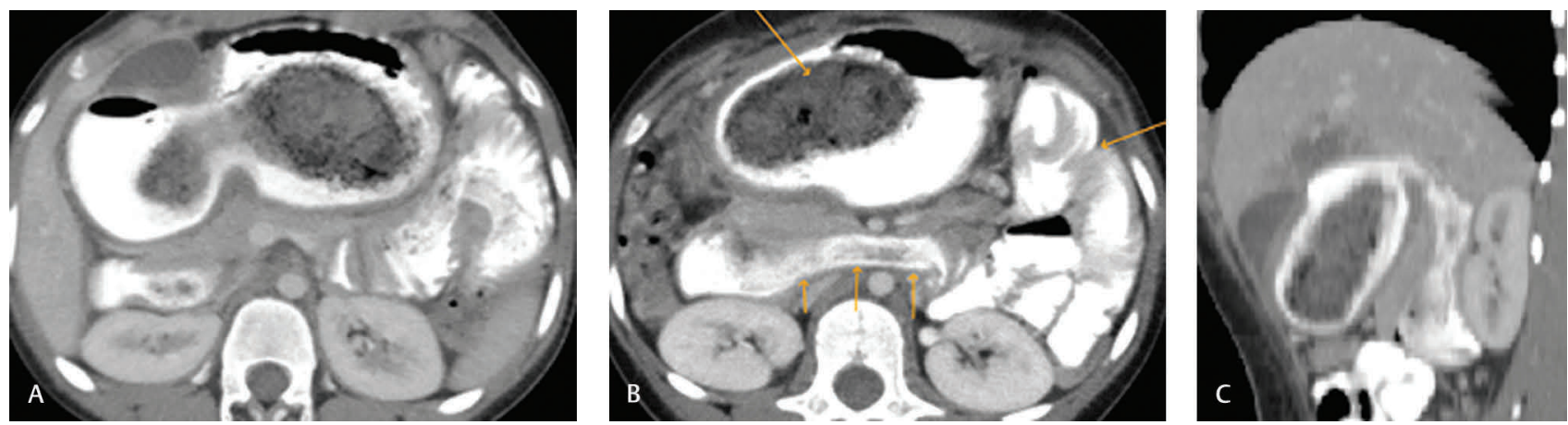

Fig. 2 Axial computed tomography (CT) with oral and intravenous contrast depicts the bezoar, seen as a nonenhancing heterogeneous intraluminal mass with mottled air foci within, coursing across the stomach, D3 segment of the duodenum, and proximal jejunum (arrows). A bezoar may occasionally be confused for ingested food material. 
small intestine like a tail, it is referred to as the Rapunzel syndrome, a rare form of trichobezoar. ${ }^{1}$ Rapunzel syndrome has usually been described in association with ingested human hair. There have only been a few case reports of cotton thread bezoar reported in the literature, ${ }^{3,4}$ and fewer reported in association with Rapunzel syndrome., ${ }^{2,6}$ It has been postulated that smooth strands of hair or organic fibers that fail to propel distally by gastric peristalsis are chronically lodged in the gastric mucosal folds, and are molded into a ball by peristaltic activity and pyloric hold-up to form a bezoar. Finally, resultant gastric atony leads to retention of the bezoar in the stomach. ${ }^{6,11}$ Gastric hypomotility and surgical procedures such as vagotomy have also been implicated as predisposing factors. ${ }^{4}$

Bezoars may be asymptomatic for a long time, or present with nausea, vomiting, abdominal pain, dyspepsia, or GI bleeding. If undiagnosed, they may lead to ulceration, perforation, peritonitis, pancreatitis, biliary obstruction, nutritional disturbances such as protein-losing enteropathy, anemia, and vitamin B12 deficiency, and, rarely, intestinal obstruction. ${ }^{6}$

Intussusception, as a complication of bezoar, has also been rarely described in the literature. Ha et al reported a case of multiple small bowel intussusceptions and bowel obstruction caused by a gastric trichobezoar in a 5-year-old girl, subsequently reduced by laparoscopic intervention. ${ }^{7}$ Prasanna et al reported a case of Rapunzel syndrome from trichobezoar, with multiple small intestinal intussusceptions which were manually reduced at surgery. ${ }^{8}$ Anantha et al reported a similar case in a 9-year-old boy, who had a gastrostomy and multiple enterotomies to retrieve a thick bezoar causing long segment of ileal intussusception. ${ }^{9}$

Although a bezoar is not inherently attached to the bowel wall, it seems to incite small bowel intussusception. It has been postulated that the tail of the bezoar and the intraluminal strands interfere with small bowel peristalsis; the resultant bowel dysmotility and mucosal irritation possibly predisposes to intussusception. ${ }^{9}$ Another theory for the etiology of intussusception, explained by Naik et al, is a pursestring effect, due to shorter length of the bezoar tail, compared with the length of the small intestine, leading to foreshortening or contraction of the mesenteric side of the small bowel wall. ${ }^{10}$ Whatever the cause, it is important to remember that bezoars may sometimes be the undeclared cause of intussusception, especially in recurrent cases. In our case, however, the intussusceptions did not cause bowel obstruction and were assumed to be transient and self-limiting.

Imaging is crucial in the diagnosis of bezoar. Abdominal radiographs may demonstrate a mottled mass in the stomach. Ultrasonography may depict a curvilinear hyperechoic mass with bright echogenic bands caused by the fibers. However, multiple acoustic interfaces created by trapped air and ingested food as well as posterior acoustic shadowing may limit adequate visualization of the mass. ${ }^{1,12}$ On an upper GI contrast study, an intraluminal mass having no connection to the gastric wall, with "barium in the honeycomb interstices" has been described. It also helps delineate ulcers that may occur as a result of the bezoar. Fluoroscopic contrast study with the patient in the erect position may demonstrate initial contrast stasis, followed by slow insinuation on all sides of an intraluminal mass, outlining the curvatures and contour of the stomach, like a cast. ${ }^{10} \mathrm{CT}$ may demonstrate a nonenhancing heterogeneous intraluminal mass, with mottled air foci within, and a sign described as "compressed concentric rings." ${ }^{13}$ The extent of the bezoar and associated complications can be assessed on CT. A bezoar may be mistaken for retained food debris due to a gastric outlet obstruction; however, the lack of a straight air-fluid-debris level and the presence of oral contrast or gastric fluid insinuating between the dependent gastric wall and the surface of the bezoar can help differentiate these entities. Sometimes, a high index of clinical suspicion may be required for diagnosis. ${ }^{7}$ Upper GI endoscopy is the investigation of choice in these patients and may also aid therapeutic retrieval of the bezoar; however, this may not be achievable in all patients, especially those with large bezoars, and does carry a small risk of perforation. ${ }^{1}$

Surgical removal is the treatment of choice for Rapunzel syndrome to prevent complications due to the enormous length of the bezoar..$^{14}$ It is important to keep in mind that multiple bezoars may be lodged distally in the small intestine, and must be excluded at preoperative imaging and intraoperative examination. ${ }^{2}$ Needless to say, adequate psychological support and counseling following removal of the bezoar, along with parental support and supervision, is crucial to prevent recurrence.

In summary, Rapunzel syndrome due to a cotton thread bezoar is rare, and a high index of suspicion and familiarity with imaging features is important to prevent late diagnosis and complications. Rapunzel syndrome may sometimes be a rare cause of small bowel intussusception with or without bowel obstruction. It is important to remember that intussusception in a child or adult may be triggered by ingested foreign bodies.

"Rapunzel, Rapunzel, let down your hair, so that I may climb thy golden stair," so said the Brothers Grimm, Jacob and Wilhelm Grimm, German philologists, cultural researchers, and lexicographers, the creators of the interesting Rapunzel story.

\section{Conflict of Interest}

None.

\section{References}

1 Jain M, Solanki SL, Bhatnagar A, Jain PK. An unusual case report of Rapunzel syndrome trichobezoar in a 3-year-old boy. Int J Trichology 2011;3(2):102-104

2 Emre AU, Tascilar O, Karadeniz G, Irkorucu O, Karakaya K, Comert M. Rapunzel syndrome of a cotton bezoar in a multimorbid patient. Clinics (Sao Paulo) 2008;63(2):285-288

3 Sethi P, Ujawal S. Hair-cotton threads bezoar in two years child. J Coll Physicians Surg Pak 2014;24 (Suppl 3):S265-S266

4 Chintamani DR, Durkhure R, Singh JP, Singhal V. Cotton bezoar--a rare cause of intestinal obstruction: case report. BMC Surg 2003;3:5

5 Singh A, Sharma AK, Choudhary HR, Moharjal P, Choudhary DK. Atypical jejunal perforation due to cotton threads bezoar in a postpartum woman associated with pica. J Clin Diagn Res 2016;10(5):PL01-PL02

6 Gonuguntla V, Joshi D-D. Rapunzel syndrome: a comprehensive review of an unusual case of trichobezoar. Clin Med Res 2009;7(3):99-102 
7 Ha S-C, Koh C-C, Lee C-H. A rare case of Rapunzel syndrome with multiple small bowel intussusceptions and bowel obstruction. Formosan J Surg 2016;49(2):67-69

8 Prasanna BK, Sasikumar K, Gurunandan U, Sreenath GS, Kate V. Rapunzel syndrome: a rare presentation with multiple small intestinal intussusceptions. World J Gastrointest Surg 2013;5(10):282-284

9 Anantha RV, Chadi SA, Merritt N. Trichobezoar causing intussusception: youngest case of Rapunzel syndrome in a boy in North America. J Pediatr Surg Case Rep 2013;1:e11-e13

10 Naik S, Gupta V, Naik S, et al. Rapunzel syndrome reviewed and redefined. Dig Surg 2007;24(3):157-161
11 Deslypere JP, Praet M, Verdonk G. An unusual case of the trichobezoar: the Rapunzel syndrome. Am J Gastroenterol 1982;77(7):467-470

12 Dhinakar M, Balkhair W. Rapunzel syndrome: a case report. Oman Med J 2010;25(4):e016

13 Lalith S, Gopalakrishnan KL, Ilangovan G, Jayajothi A. Rapunzel syndrome. J Clin Diagn Res 2017;11(9):TD01-TD02

14 Phillips MR, Zaheer S, Drugas GT. Gastric trichobezoar: case report and literature review. Mayo Clin Proc 1998;73(7):653-656 